\title{
Cryo-EM strikes gold
}

Gold supports nearly eliminate unwanted specimen motion in cryo-electron microscopy, enabling highresolution structure determination of challenging proteins.

The field of single-particle cryo-electron microscopy (cryo-EM) has made amazing technical strides in the past few years. In particular, the recent development of highly sensitive direct electron detectors are allowing cryo-EM to reach what was previously the realm of crystallography: atomic-resolution structure determination, but without the requirement of protein crystallization.

But even researchers using direct electron detectors still

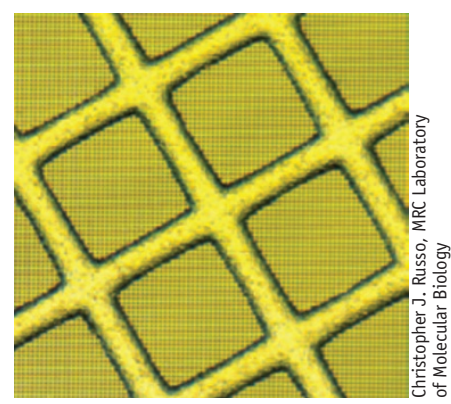

Gold specimen supports improve cryo-EM image quality. encounter the pesky problem of the specimen support moving in the electron beam. In a typical cryo-EM experiment, protein particles are frozen in ice suspended across tiny holes in an amorphous carbon film supported by a metal grid. For reasons that are not fully understood, but likely because of certain instabilities in the carbon film, the specimen support undergoes deformation when irradiated by the electron beam. This causes the captured images to blur and limits researchers' ability to align the particle images to achieve high-resolution structure determination.

Lori Passmore and her postdoctoral fellow Christopher Russo of the Medical Research Council (MRC) Laboratory of Molecular Biology have been interested in addressing cryo-EM specimen motion at the root of the problem: the support itself. In the June 2014 issue of Nature Methods, they reported that a hydrogen plasma-treated graphene support was subject to substantially less electron beam-induced motion than the conventional amor-

\section{GENOMICS}

\section{CRISPR GAIN-OF-FUNCTION SCREENS}

An engineered dCas9-guide RNA complex containing three different transcriptional activation domains achieves consistently high expression of endogenous genes in a genome-wide screen.

Important insights into synergistic gene function can be gained either by eliminating genes and studying the effect of loss of function or by overexpressing genes to observe the consequences of gains in function. Loss-of-function screens have been reported using a variety of methods, from RNA interference to CRISPR (clustered, regularly interspaced, short palindromic repeats) screens, but to increase the expression of genes on a genome-wide scale is more challenging. Feng Zhang and his team at the Broad Institute of MIT and Harvard in Boston recently adapted the CRISPR system for this purpose.

The guide RNA (gRNA) that brings the Cas9 nuclease, or a catalytically inactive version (dCas9), to its complementary target sequence in the genome has seen a multitude of applications, including gene activation. dCas9 fused to a transcriptional activator (such as VP64) can be directed to an endogenous locus and upregulates the gene. This approach, however, has proven more difficult to multiplex than initially anticipated. It often takes multiple gRNA-dCas9-activator complexes to achieve activation, and the increase in expression can be small. "The challenge with dCas9VP64 alone is that, for many genes, it fails to achieve activation using a single guide RNA," recalls Zhang. "Therefore, it was not possible to develop a pooled activation screening method using individual guide RNAs and dCas9-VP64." Instead, his group's goal was to "develop a system where any gene we target has a high probability of being activated."

Zhang, together with graduate student Silvana Konermann, decided to start with the atomic structure of Cas 9 to rationally engineer a potent transcription activation complex. "0therwise," explains Zhang, "if we designed a screen using a 60,000-guide 
phous carbon support. Moreover, they found that a continuous layer of graphene allowed much more optimal control of protein distribution on the support surface than a continuous layer of amorphous carbon.

Now, reporting in Science, Passmore and Russo show that gold is even better than graphene at reducing specimen motion, to the point that such movement is nearly eradicated. Compared to a standard amorphous carbon support, their use of gold reduced motion of the specimen by 50-fold, which translates to a twofold reduction in image blurring. The gold support allowed them to solve the structure of the iron-storage protein apoferritin, a small, spherical protein that has presented challenges for cryo-EM structure determination, at 4.7-angstrom resolution.

The gold support consists of an array of micrometer-sized holes in a thin gold foil, which is suspended across a mesh grid also made of gold. "Chris chose gold because it is compatible with biological specimens, it is conductive, stable (non-oxidizing) and radiation hard," says Passmore. Although gold supports should be relatively straightforward for many labs to manufacture themselves, Passmore and Russo are also working with a company to commercialize them. Passmore further points out that gold supports have the potential to improve images from any electron microscope, not only those equipped with the latest-generation detectors.

Passmore and Russo are interested to next explore whether combining the advantages of a continuous graphene layer with the gold support will even further improve protein structure determination by cryo-EM. "Graphene addresses the problem of providing a reproducible, invisible and tunable surface for the adsorption of proteins. Gold supports virtually eliminate the motion of the specimen support during irradiation," Russo explains. "Taken together, these address two major problems in cryo-EM."

\section{Allison Doerr}

\section{RESEARCH PAPERS}

Russo, C.J. \& Passmore, L.A. Controlling protein adsorption on graphene for cryo-EM using low-energy hydrogen plasmas. Nat. Methods 11, 649-652 (2014).

Russo, C.J. \& Passmore, L.A. Ultrastable gold substrates for electron cryomicroscopy. Science 346, 1377-1380 (2014).

RNA library where many of the guides are not able to activate the target gene, we would not be very confident that the screen probed most of the genes we tried to target."

The first question was where in the Cas9-gRNA complex to attach the activating domains. They decided on two regions in the gRNA that protrude out of the complex and whose deletions did not affect function; they then added a small aptamer that binds the bacteriophage coat protein MS2. This aptamer recruits activation domainMS2 fusions. After optimizing various combinations, the team settled on MS2 fused to p65 and HSF1, the activation domains of NF- $\mathrm{KB}$ and heat-shock factor 1, respectively, and recruited these to a gRNA bound to a dCas9-VP64 fusion. This transcription activation system, termed SAM for synergistic activation mediator, consisted of three different activation domains in the CRISPR complex.

When testing SAM with about 100 gRNAs on 12 endogenous genes in a human cell line, the researchers achieved optimal activation when the gRNAs bound no more than 200 base pairs upstream of the transcription start site. Upregulation was not confined to proteincoding genes: SAM also achieved high expression levels of long noncoding RNAs and showed no appreciable off-target activity. The system was thus ready for a genome-wide screen.

The researchers designed $\sim 70,000$ gRNAs targeting the 23,430 coding gene isoforms found in the NCBI RefSeq database, cloned them into lentiviral vectors and transfected a human melanoma cell line that expressed dCas9-VP64 and MS2-p65HSF1 fusion proteins. After 7 days of selection, an inhibitor to the oncogene BRAF was added, and 14 days later the DNA of inhibitor-resistant cells was sequenced to determine which genes had been activated to achieve this gain of function. Their results point to known as well as previously unknown mechanisms of resistance. Zhang now plans to use SAM for a different cell type: "We are really excited to look at genes in the brain that may contribute to disease or protect from disease."

Nicole Rusk 